Bull. Korean Math. Soc. 50 (2013), No. 1, pp. 97-103

http://dx.doi.org/10.4134/BKMS.2013.50.1.097

\title{
RIGIDITY THEOREMS IN THE HYPERBOLIC SPACE
}

\author{
Henrique FERnANDES DE Lima
}

\begin{abstract}
As a suitable application of the well known generalized maximum principle of Omori-Yau, we obtain rigidity results concerning to a complete hypersurface immersed with bounded mean curvature in the $(n+1)$-dimensional hyperbolic space $\mathbb{H}^{n+1}$. In our approach, we explore the existence of a natural duality between $\mathbb{H}^{n+1}$ and the half $\mathcal{H}^{n+1}$ of the de Sitter space $\mathbb{S}_{1}^{n+1}$, which models the so-called steady state space.
\end{abstract}

\section{Introduction}

In this paper, we are interested in the study of complete non-compact hypersurfaces immersed with bounded mean curvature in the $(n+1)$-dimensional hyperbolic space $\mathbb{H}^{n+1}$. Before giving details on our work, we present a brief outline of the main results related to our ones.

In [1], L. J. Alías and M. Dajczer studied complete surfaces properly immersed in $\mathbb{H}^{3}$ which are contained between two horospheres, obtaining a Bernstein-type result for the case of constant mean curvature $-1 \leq H \leq 1$.

The author and A. Caminha have studied in [3] complete vertical graphs of constant mean curvature in $\mathbb{H}^{n+1}$. Under appropriate restriction on the growth of the height function, they obtained necessary conditions for the existence of such a graph. Furthermore, for complete surfaces of nonnegative Gaussian curvature, they obtained a Bernstein-type theorem in $\mathbb{H}^{3}$.

More recently, by applying a technique of S. T. Yau [13], the author jointly with F. E. C. Camargo and A. Caminha [2] have also obtained Bernstein-type results in $\mathbb{H}^{n+1}$.

Here, under an appropriated restriction on the normal angle of the hypersurface (that is, the angle between the Gauss map of the hypersurface and the unitary vector field which determines on $\mathbb{H}^{n+1}$ a codimension one foliation by horospheres; see Section 3), we obtain rigidity theorems concerning to a complete hypersurface immersed with bounded mean curvature in $\mathbb{H}^{n+1}$. In our approach, we explore the existence of a natural duality between $\mathbb{H}^{n+1}$ and the

Received May 4, 2011.

2010 Mathematics Subject Classification. Primary 53C42; Secondary 53B30, 53C50.

Key words and phrases. hyperbolic space, complete hypersurfaces, mean curvature, Gauss map.

The author was partially supported by FAPESQ/CNPq/PPP, Brazil. 
half $\mathcal{H}^{n+1}$ of the de Sitter space $\mathbb{S}_{1}^{n+1}$, which models the so-called steady state space (cf. Sections 2 and 3 ).

We prove the following (cf. Theorem 3.3; see also Corollaries 3.5 and 3.6):

Let $\psi: \Sigma^{n} \rightarrow \mathbb{H}^{n+1}$ be a complete hypersurface, with bounded second fundamental form A. Suppose that the (not necessarily constant) mean curvature $H$ of $\Sigma^{n}$ is such that $0 \leq H \leq 1$. If $\Sigma^{n}$ is under a horosphere of $\mathbb{H}^{n+1}$ and its normal angle $\theta$ satisfies $\cos \theta \geq \sup _{\Sigma} H$, then $\Sigma^{n}$ is a horosphere and the image of its Lorentz Gauss map is exactly a hyperplane of $\mathcal{H}^{n+1}$.

We want to point out that our restriction on the normal angle of the hypersurface is motivated by a gradient estimate due to R. López and S. Montiel [6] (for more details, see Remark 3.4).

Furthermore, by applying a classical result due to A. Huber [4] concerned with parabolic surfaces, we also prove the following (cf. Theorem 3.7):

Let $\psi: \Sigma^{2} \rightarrow \mathbb{H}^{3}$ be a complete surface of nonnegative Gaussian curvature and with (not necessarily constant) mean curvature $0 \leq H \leq 1$. If the normal angle $\theta$ of $\Sigma^{2}$ satisfies $\cos \theta \geq H$, then $\Sigma^{2}$ is a horosphere and the image of its Lorentz Gauss map is exactly a plane of $\mathcal{H}^{3}$.

\section{The steady state space $\mathcal{H}^{n+1}$}

In order to study the geometry of the Gauss map of a hypersurface immersed in the hyperbolic space, we need some preliminaries of Lorentz geometry.

Let $\mathbb{L}^{n+2}$ denote the $(n+2)$-dimensional Lorentz-Minkowski space $(n \geq 2)$, that is, the real vector space $\mathbb{R}^{n+2}$ endowed with the Lorentz metric defined by

$$
\langle v, w\rangle=\sum_{i=1}^{n+1} v_{i} w_{i}-v_{n+2} w_{n+2}
$$

for all $v, w \in \mathbb{R}^{n+2}$. We define the $(n+1)$-dimensional de Sitter space $\mathbb{S}_{1}^{n+1}$ as the following hyperquadric of $\mathbb{L}^{n+2}$ :

$$
\mathbb{S}_{1}^{n+1}=\left\{p \in L^{n+2} ;\langle p, p\rangle=1\right\} .
$$

The induced metric from $\langle$,$\rangle makes \mathbb{S}_{1}^{n+1}$ into a Lorentz manifold with constant sectional curvature one. Moreover, if $p \in \mathbb{S}_{1}^{n+1}$, we can put

$$
T_{p}\left(\mathbb{S}_{1}^{n+1}\right)=\left\{v \in \mathbb{L}^{n+2} ;\langle v, p\rangle=0\right\} .
$$

Let $a \in \mathbb{L}^{n+2}$ be a non-zero null vector in the past half of the null cone (with vertex in the origin), that is, $\langle a, a\rangle=0$ and $\left\langle a, e_{n+2}\right\rangle>0$, where $e_{n+2}=$ $(0, \ldots, 0,1)$. Then the open region of the de Sitter space $\mathbb{S}_{1}^{n+1}$, given by

$$
\mathcal{H}^{n+1}=\left\{x \in \mathbb{S}_{1}^{n+1} ;\langle x, a\rangle>0\right\}
$$

is the so-called steady state space. Observe that $\mathcal{H}^{n+1}$ is extendible and, so, non-compact, being only half a de Sitter space. Its boundary, as a subset of $\mathbb{S}_{1}^{n+1}$, is the null hypersurface

$$
\left\{x \in \mathbb{S}_{1}^{n+1} ;\langle x, a\rangle=0\right\},
$$


whose topology is that of $\mathbb{R} \times \mathbb{S}^{n-1}$ (cf. [7]).

Now, we shall consider in $\mathcal{H}^{n+1}$ the timelike field

$$
\mathcal{K}=\langle x, a\rangle x-a .
$$

We easily see that

$$
\bar{\nabla}_{V} \mathcal{K}=\langle x, a\rangle V \text { for all } V \in \mathfrak{X}\left(\mathcal{H}^{n+1}\right),
$$

that is, $\mathcal{K}$ is closed and conformal field on $\mathcal{H}^{n+1}$ (cf. [5], Section 2). Then, from Proposition 1 of [9], we have that the $n$-dimensional distribution $\mathcal{D}$ defined on $\mathcal{H}^{n+1}$ by

$$
p \in \mathcal{H}^{n+1} \longmapsto \mathcal{D}(p)=\left\{v \in T_{p} \mathcal{H}^{n+1} ;\langle\mathcal{K}(p), v\rangle=0\right\}
$$

determines a codimension one spacelike foliation $\mathcal{F}(\mathcal{K})$ which is oriented by $\mathcal{K}$. Moreover (cf. [10], Example 1), the leaves of $\mathcal{F}(\mathcal{K})$ are hyperplanes

$$
\mathcal{L}_{\rho}=\left\{x \in \mathbb{S}_{1}^{n+1} ;\langle x, a\rangle=\rho\right\}, \rho>0,
$$

which are totally umbilical hypersurfaces of $\mathcal{H}^{n+1}$ isometric to the Euclidean space $\mathbb{R}^{n}$, and having constant mean curvature 1 with respect to the unit pastdirected normal fields

$$
\eta_{\rho}(x)=x-\frac{1}{\rho} a, \quad x \in \mathcal{L}_{\rho} .
$$

\section{Rigidity results in $\mathbb{H}^{n+1}$}

In this section, instead of the more commonly used half-space model for the $(n+1)$-dimensional hyperbolic space, we consider the warped product model

$$
\mathbb{H}^{n+1}=\mathbb{R} \times e^{t} \mathbb{R}^{n} .
$$

It can easily be seen that the fibers $M_{t_{0}}=\left\{t_{0}\right\} \times \mathbb{R}^{n}$ of the warped product model are precisely the horospheres of $\mathbb{H}^{n+1}$. Moreover, these have constant mean curvature 1 if we take the orientation given by the unit normal vector field $N=-\partial_{t}$ (cf. [8], Example 3 of Section 4).

Another useful model for $\mathbb{H}^{n+1}$ is the so-called Lorentz model, obtained by furnishing the hyperquadric

$$
\left\{p \in \mathbb{L}^{n+2} ;\langle p, p\rangle=-1, p_{n+2}>0\right\}
$$

with the (Riemannian) metric induced by the Lorentz metric of $\mathbb{L}^{n+2}$. In this setting, if $a \in \mathbb{L}^{n+2}$ denotes a fixed null vector as in the beginning of the previous section, a typical horosphere is

$$
L_{\tau}=\left\{p \in \mathbb{H}^{n+1} ;\langle p, a\rangle=\tau\right\},
$$

where $\tau$ is a positive real number. A straightforward computation shows that

$$
\xi_{p}=-p-\frac{1}{\tau} a \in \mathcal{H}^{n+1}
$$

is a unit normal vector field along $L_{\tau}$, with respect to which $L_{\tau}$ has mean curvature 1 (cf. [6], Section 3). 
In the context of the Lorentz model of $\mathbb{H}^{n+1}$, we say that a hypersurface $\psi: \Sigma^{n} \rightarrow \mathbb{H}^{n+1}$ is under a horosphere $L_{\tau}$ when $\langle\psi, a\rangle \leq \tau$. In this case, if we consider the warped model of $\mathbb{H}^{n+1}$, we easily see that the height function $h=\pi_{\mathbb{R}} \circ \psi$ of $\Sigma^{n}$ is bounded from above.

Now, we present our analytical framework.

Lemma 3.1 ([3], Proposition 3.2). Let $\psi: \Sigma^{n} \rightarrow \mathbb{R} \times_{f} M^{n}$ be a hypersurface immersed into a Riemannian warped product $\mathbb{R} \times_{f} M^{n}$, with Gauss map $N$. Then, by denoting $h=\pi_{I} \circ \psi$ the height function of $\Sigma^{n}$, we have

$$
\Delta h=(\ln f)^{\prime}(h)\left(n-|\nabla h|^{2}\right)+n H\left\langle N, \partial_{t}\right\rangle .
$$

We also will need the well known generalized Maximum Principle due to H. Omori and S. T. Yau [11, 12].

Lemma 3.2. Let $\Sigma^{n}$ be an $n$-dimensional complete Riemannian manifold whose Ricci curvature is bounded from below and $u: \Sigma^{n} \rightarrow \mathbb{R}$ be a smooth function which is bounded from above on $\Sigma^{n}$. Then there is a sequence of points $\left\{p_{k}\right\}$ in $\Sigma^{n}$ such that

$$
\lim _{k \rightarrow \infty} u\left(p_{k}\right)=\sup _{\Sigma} u, \lim _{k \rightarrow \infty}\left|\nabla u\left(p_{k}\right)\right|=0 \text { and } \lim _{k \rightarrow \infty} \Delta u\left(p_{k}\right) \leq 0 .
$$

In what follows, we will consider an isometry $\Phi$ between the warped product and Lorentz models of $\mathbb{H}^{n+1}$ which carries $\left(\partial_{t}\right)_{q}$ to $\Phi_{*}\left(\partial_{t}\right)=\xi_{\Phi(q)}$ (such isometry is given in [1]). In this setting, it is natural to consider the Lorentz Gauss map of $\Sigma$ with respect to $N$ as given by

$$
\begin{array}{ccc}
\Sigma^{n} & \rightarrow & \mathcal{H}^{n+1} \\
p & \mapsto & -\Phi_{*}\left(N_{p}\right) .
\end{array}
$$

Given a hypersurface $\Sigma^{n}$ in $\mathbb{H}^{n+1}$ whose Gauss map satisfies $\left\langle N, \partial_{t}\right\rangle<0$, we define the normal angle $\theta$ of $\Sigma^{n}$ as being the smooth function $\theta: \Sigma^{n} \rightarrow\left[0, \frac{\pi}{2}\right]$ given by

$$
0 \leq \cos \theta=-\left\langle N, \partial_{t}\right\rangle \leq 1
$$

Now, we can state and prove our main result.

Theorem 3.3. Let $\psi: \Sigma^{n} \rightarrow \mathbb{H}^{n+1}$ be a complete hypersurface, with bounded second fundamental form $A$. Suppose that the mean curvature $H$ of $\Sigma^{n}$ is such that $0 \leq H \leq 1$. If $\Sigma^{n}$ is under a horosphere of $\mathbb{H}^{n+1}$ and its normal angle $\theta$ satisfies $\cos \theta \geq \sup _{\Sigma} H$, then $\Sigma^{n}$ is a horosphere and the image of its Lorentz Gauss map is exactly a hyperplane of $\mathcal{H}^{n+1}$.

Proof. Initially, let us consider $X \in \mathfrak{X}(\Sigma)$ with $|X|=1$. It follows from Gauss equation that

$$
\operatorname{Ric}_{\Sigma}(X)=1-n+n H\langle A X, X\rangle-\langle A X, A X\rangle,
$$

where $\operatorname{Ric}_{\Sigma}$ stands for the Ricci curvature of $\Sigma^{n}$. Hence,

$$
\operatorname{Ric}_{\Sigma} \geq 1-n-n H|A|-|A|^{2} .
$$


Thus, since $H$ and $A$ are supposed to be bounded, we conclude that $\mathrm{Ric}_{\Sigma}$ is bounded from below on $\Sigma^{n}$.

Now, from Lemma 3.1, we have that

$$
\Delta h=n\left(1+H\left\langle N, \partial_{t}\right\rangle\right)-|\nabla h|^{2} .
$$

On the other hand, since $\Sigma^{n}$ is supposed to be under a horosphere of $\mathbb{H}^{n+1}$ and its Ricci curvature is bounded from below, we are in position to apply Lemma 3.2 to the function $h$, obtaining a sequence $\left\{p_{k}\right\}$ in $\Sigma^{n}$ such that

$$
\lim _{k \rightarrow \infty} h\left(p_{k}\right)=\sup _{\Sigma} h, \lim _{k \rightarrow \infty}\left|\nabla h\left(p_{k}\right)\right|=0 \text { and } \lim _{k \rightarrow \infty} \Delta h\left(p_{k}\right) \leq 0 .
$$

Consequently, since the functions $H$ and $\left\langle N, \partial_{t}\right\rangle$ are bounded on $\Sigma^{n}$, we get a subsequence $\left\{p_{k_{j}}\right\}$ of $\left\{p_{k}\right\}$ such that

$$
0 \geq \lim _{j \rightarrow \infty} \Delta h\left(p_{k_{j}}\right) \geq n\left(1-\lim _{j \rightarrow \infty} H\left(p_{k_{j}}\right)\right) \geq 0 .
$$

Then, $\lim _{j \rightarrow \infty} H\left(p_{k_{j}}\right)=1$, and $\sup _{\Sigma} H=1$. Thus, since we are supposing that the normal angle $\theta$ of $\Sigma^{n}$ satisfies $\cos \theta \geq \sup _{\Sigma} H$, we get that $\left\langle N, \partial_{t}\right\rangle=$ $-\cos \theta=-1$ on $\Sigma^{n}$ and, hence, $\Sigma^{n}$ is a horosphere. Moreover, by considering an isometry $\Phi$ between the warped product and Lorentz models of $\mathbb{H}^{n+1}$, we get

$$
\langle N, a\rangle=\left\langle-\partial_{t}, a\right\rangle=\left\langle-\xi_{\Phi}, a\right\rangle=\langle\psi, a\rangle
$$

and, therefore, we conclude that $N(\Sigma)$ is exactly a hyperplane of $\mathcal{H}^{n+1}$.

Remark 3.4. Let $\psi: \Sigma^{n} \rightarrow \mathbb{H}^{n+1}$ be a immersion from a compact manifold $\Sigma^{n}$ with mean convex boundary $\partial \Sigma$ contained into a horosphere $L_{\tau}$, for some $\tau>0$. Suppose that $\psi$ has constant mean curvature $0 \leq H \leq 1$. From the gradient estimate (19) of [6], taking into account our choice of the orientation $N$ of $\Sigma^{n}$, we get

$$
\langle N, a\rangle \geq H \tau .
$$

Consequently, by supposing that $\Sigma^{n}$ is under the horosphere $L_{\tau}$, we conclude that its normal angle $\theta$ satisfies

$$
\cos \theta=-\left\langle N, \partial_{t}\right\rangle=\frac{1}{\langle\psi, a\rangle}\langle N, a\rangle \geq \frac{1}{\tau}\langle N, a\rangle \geq H .
$$

Since for a hypersurface $\Sigma^{n}$ immersed in $\mathbb{H}^{n+1}$ we have that

$$
|A|^{2}=n^{2} H^{2}-n(n-1)(R+1)
$$

where $R$ denotes de scalar curvature of $\Sigma^{n}$, we get:

Corollary 3.5. Let $\psi: \Sigma^{n} \rightarrow \mathbb{H}^{n+1}$ be a complete hypersurface, with scalar curvature $R$ bounded from below. Suppose that the mean curvature $H$ of $\Sigma^{n}$ is such that $0 \leq H \leq 1$. If $\Sigma^{n}$ is under a horosphere of $\mathbb{H}^{n+1}$ and its normal angle $\theta$ satisfies $\cos \theta \geq \sup _{\Sigma} H$, then $\Sigma^{n}$ is a horosphere and the image of its Lorentz Gauss map is exactly a hyperplane of $\mathcal{H}^{n+1}$. 
By using once more the existence of a natural duality between $\mathbb{H}^{n+1}$ and $\mathcal{H}^{n+1}$, we obtain the following consequence of Theorem 3.3.

Corollary 3.6. Let $\psi: \Sigma^{n} \rightarrow \mathbb{H}^{n+1}$ be a complete hypersurface, with bounded second fundamental form A. Suppose that the mean curvature $H$ of $\Sigma^{n}$ is such that $0 \leq H \leq 1$. If $\Sigma^{n}$ is under a horosphere $L_{\tau}$ and the image of its Lorentz Gauss map $N(\Sigma)$ is contained in the closure of the interior domain enclosed by a hyperplane $\mathcal{L}_{\rho}$ of $\mathcal{H}^{n+1}$, with $\frac{\rho}{\tau} \geq \sup _{\Sigma} H$, then $\Sigma^{n}$ is a horosphere and the image of its Lorentz Gauss map is exactly a hyperplane of $\mathcal{H}^{n+1}$.

Proof. By considering again an isometry $\Phi$ between the warped product and Lorentz models of $\mathbb{H}^{n+1}$, we get

$$
\left\langle N, \partial_{t}\right\rangle=\left\langle N,-\psi-\frac{1}{\langle\psi, a\rangle} a\right\rangle=-\frac{1}{\langle\psi, a\rangle}\langle N, a\rangle .
$$

Consequently, since we are supposing that $\Sigma^{n}$ is under the horosphere $L_{\tau}$ and that its Lorentz Gauss map $N(\Sigma)$ is contained in the closure of the interior domain enclosed by the hyperplane $\mathcal{L}_{\rho}$,

$$
\cos \theta=-\left\langle N, \partial_{t}\right\rangle \geq \frac{\rho}{\tau}
$$

Therefore, our hypothesis on the image of the Lorentz Gauss map of $\Sigma^{n}$ amounts to

$$
\cos \theta \geq \sup _{\Sigma} H
$$

and, hence, the result follows from Theorem 3.3.

In the 3-dimensional case, we obtain the following rigidity result concerning to complete surfaces of nonnegative Gaussian curvature.

Theorem 3.7. Let $\psi: \Sigma^{2} \rightarrow \mathbb{H}^{3}$ be a complete surface of nonnegative Gaussian curvature and with mean curvature $0 \leq H \leq 1$. If the normal angle $\theta$ of $\Sigma^{2}$ satisfies $\cos \theta \geq H$, then $\Sigma^{2}$ is a horosphere and the image of its Lorentz Gauss map is exactly a plane of $\mathcal{H}^{3}$.

Proof. By applying Lemma 3.1, we get

$$
\begin{aligned}
\Delta e^{-h} & =e^{-h}\left(|\nabla h|^{2}-\Delta h\right) \\
& =2 e^{-h}\left(|\nabla h|^{2}-1-H\left\langle N, \partial_{t}\right\rangle\right) .
\end{aligned}
$$

On the other hand, since $h=\pi_{\mathbb{R}_{\mid \Sigma}}$, one has

$$
\begin{aligned}
\nabla h & =\nabla\left(\pi_{\mathbb{R}_{\mid \Sigma}}\right)=\left(\bar{\nabla} \pi_{\mathbb{R}}\right)^{\top}=\partial_{t}^{\top} \\
& =\partial_{t}-\left\langle N, \partial_{t}\right\rangle N,
\end{aligned}
$$

where $\bar{\nabla}$ denotes the gradient with respect to the metric of $\mathbb{H}^{3}$, and ()$^{\top}$ the tangential component of a vector field in $\mathfrak{X}\left(\mathbb{H}^{3}\right)$ along $\Sigma^{2}$. Consequently, $|\nabla h|^{2}=1-\cos ^{2} \theta$. Thus,

$$
\Delta e^{-h}=2 e^{-h} \cos \theta(H-\cos \theta)
$$


and, hence, our hypothesis on the normal angle $\theta$ of $\Sigma^{2}$ guarantees that the function $e^{-h}$ is a superharmonic positive function on $\Sigma$. However, a classical result due to A. Huber [4] assures that complete surfaces of nonnegative Gaussian curvature must be parabolic. Therefore, $h$ is constant on $\Sigma^{2}$, that is, $\Sigma^{2}$ is a horosphere of $\mathbb{H}^{3}$ and the image of its Lorentz Gauss map $N(\Sigma)$ is exactly a plane of $\mathcal{H}^{3}$.

\section{References}

[1] L. J. Alías and M. Dajczer, Uniqueness of constant mean curvature surfaces properly immersed in a slab, Comment. Math. Helv. 81 (2006), no. 3, 653-663.

[2] F. E. C. Camargo, A. Caminha, and H. F. de Lima, Bernstein-type Theorems in SemiRiemannian Warped Products, Proc. Amer. Math. Soc. 139 (2011), no. 5, 1841-1850.

[3] A. Caminha and H. F. de Lima, Complete vertical graphs with constant mean curvature in semi-Riemannian warped products, Bull. Belg. Math. Soc. Simon Stevin 16 (2009), no. 1, 91-105.

[4] A. Huber, On subharmonic functions and differential geometry in the large, Comment. Math. Helv. 32 (1957), 13-72.

[5] H. F. de Lima, Spacelike hypersurfaces with constant higher order mean curvature in de Sitter space, J. Geom. Phys. 57 (2007), no. 3, 967-975.

[6] R. López and S. Montiel, Existence of constant mean curvature graphs in hyperbolic space, Calc. Var. Partial Differential Equations 8 (1999), no. 2, 177-190.

[7] S. Montiel, Complete non-compact spacelike hypersurfaces of constant mean curvature in de Sitter spaces, J. Math. Soc. Japan 55 (2003), no. 4, 915-938.

[8] Unicity of constant mean curvature hypersurfaces in some Riemannian manifolds, Indiana Univ. Math. J. 48 (1999), no. 2, 711-748.

[9] Uniqueness of spacelike hypersurfaces of constant mean curvature in foliated spacetimes, Math. Ann. 314 (1999), no. 3, 529-553.

[10] _ An integral inequality for compact spacelike hypersurfaces in De Sitter space and applications to the case of constant mean curvature, Indiana Univ. Math. J. 37 (1988), no. 4, 909-917.

[11] H. Omori, Isometric immersions of Riemannian manifolds, J. Math. Soc. Japan 19 (1967), 205-214.

[12] S. T. Yau, Harmonic functions on complete Riemannian manifolds, Comm. Pure Appl. Math. 28 (1975), 201-228.

[13] Some function-theoretic properties of complete Riemannian manifolds and their applications to geometry, Indiana Univ. Math. J. 25 (1976), no. 7, 659-670.

Departamento de Matemática e Estatística

Universidade Federal de Campina Grande

Campina Grande, Paraíba, Brazil

E-mail address: henrique.delima@pq.cnpq.br 